\title{
The Natural Evolution of a Pigmented Spitz-Reed Nevus in a 24 Months Dermoscopic Follow Up
}

Dika Emi ${ }^{*}$ Piraccini Bianca Maria, Fanti Pier Alessandro, Neri Iria and Patrizi Annalisa

Department of Specialised Dermatology, Experimental and Diagnostic Medicine, Sant'Orsola -Malpighi Hospital, University of Bologna, Italy

*Corresponding author: Emi Dika, Dermatology, Via Massarenti 1, 40138 Bologna, Italy, Tel: +390516364150; Fax: +390516363474; E-mail: emi.dika3@unibo.it

Rec date: July 08, 2014; Acc date: July 09, 2014; Pub date: July 12, 2014

Copyright: @ $2014 \mathrm{Emi} \mathrm{D}$, et al. This is an open-access article distributed under the terms of the Creative Commons Attribution License, which permits unrestricted use, distribution, and reproduction in any medium, provided the original author and source are credited.

\begin{abstract}
The natural evolution of Spitz-Reed nevi is poorly understood, owing to the lack of studies investigating their longterm clinical evolution and the fact that most of the lesions are surgically excised. The surgical approach is indicated because of the clinical and histopathologic similarities with melanoma that may create diagnostic confusion in some cases. Hence nowadays, due to the low incidence of melanoma in children and the development of diagnostic dermoscopic criteria, a less aggressive approach to SRN may be reserved, especially in cases with typical features. We report a case of a pigmented lesion that showed gradual changes, from a Spitz Reed Nevus with typical clinical and dermoscopic features, to a common nevus.
\end{abstract}

Keywords: Spitz nevi; Reed nevi; Natural evolution; Involution; Dermoscopy

\section{Abbreviation \\ SRN: Spitz-Reed Nevi}

\section{Correspondence}

The natural evolution of Spitz-Reed nevi (SRN) is poorly understood, owing to the lack of studies investigating their long-term clinical evolution and the fact that most of the lesions are surgically excised [1,2].

The surgical approach is indicated because of the clinical and histopathologic similarities with melanoma that may create diagnostic confusion in some cases.

Hence nowadays, due to the low incidence of melanoma in children and the development of diagnostic dermoscopic criteria, a less aggressive approach to SRN may be reserved, especially in cases with typical features $[3,4]$.

We report herein a case of a pigmented lesion that showed gradual changes, from a Spitz Reed Nevus (SRN), with typical clinical and dermoscopic features, to a common nevus.

\section{Report of a Case}

A 6-year-old boy was referred by his pediatrician with a 4-mm, black, symmetric macule located on the right arm. Dermoscopically, the lesion exhibited a starburst pattern characterized by a rim of pigmented streaks at the periphery (Figure 1A). Because of the typical dermoscopic pattern suggestive of SRN, no biopsy was performed, and the boy was scheduled for a follow-up visit at six months, as suggested by other authors [1]. After 6 and 12 months (Figure 1B and 1C), the streaks were not discernible any more, and only remnants of lightbrown to gray pigmentation were still visible. At 24 months' time the lesion exhibited almost complete loss of the dermoscopic features of
SRN, presenting only delicate remnants of light brown pigmentation as the only visible feature (Figure 1D).

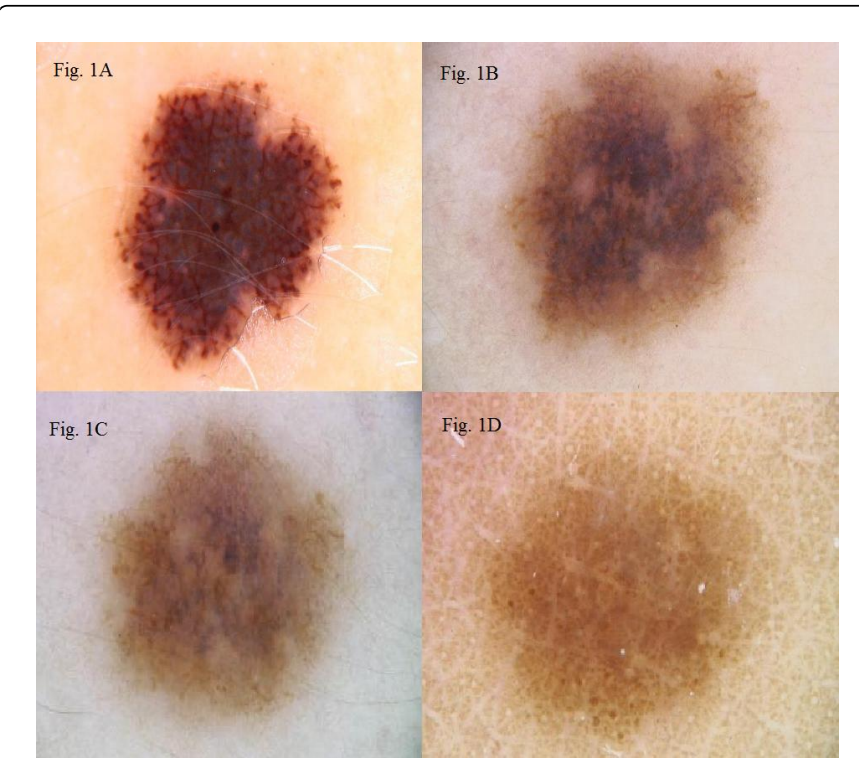

Figure 1: Dermoscopic changes of a Spitz-Reed Nevus in a 24month follow-up: starburst pattern with pigmented streaks at the periphery (Figure 1A); streaks not discernible any more (Figure 1B); then, remnants of light-brown to gray pigmentation still visible (Figure 1C); at 24 months' time, almost complete loss of dermoscopic features of Spitz-Reed nevus presenting only delicate remnants of light brown pigmentation (Figure 1D).

\section{Comment}

Spitz Reed nevi are acquired, benign, melanocytic proliferations, commonly encountered in the clinical practice. Surgical excision and histopathology were the diagnostic tools and the treatment required 
Citation: Emi D, Maria PB, Alessandro FP, Iria N, Annalisa P (2014) The Natural Evolution of a Pigmented Spitz-Reed Nevus in a 24 Months Dermoscopic Follow Up. Pigmentary Disorders 1: 119. doi:10.4172/jpd.1000119

Page 2 of 2

prior to dermoscopy. The increasing confidence of the clinicians with this non-invasive technique and the development of morphologic criteria for the diagnosis of pigmented lesions, nowadays, enables trained clinicians to identify peculiar characteristics of these lesions in order to prevent unusual excisions when possible [4,5]. In pediatric population a surgical excision may have to deal with general anesthesia and collateral effects. Most of the parents would not be happy to deal with such decisions. The incidence of melanoma in a pediatric population is extremely rare [3] and when dealing with lesions that present typical characteristic features of SRN, literature data suggest that a non-aggressive approach is now well accepted. Other authors as a matter of fact, already published their data on the natural evolution of SRN [6,7]. They emphasize the fact that the involution of SRN might be the reason why the incidence of these lesions in adults seems to be much lower than in children. Hence, precise clinical and dermoscopic criteria of SRN that go through an involution process have not been described in large series, especially in children in order to evaluate the percentage of lesions that involve, in order to make such an assumption.

Their reports together with this case we presented, strongly suggest the hypothesis that lager study on clinical evolution of SRN should be performed, in order to establish a standardized approach.

\section{References}

1. Argenziano G, Zalaudek I, Ferrara G, Lorenzoni A, Soyer HP (2007) Involution: The Natural Evolution of Pigmented Spitz and Reed. Arch Dermatol 143: 549-551.

2. Vollmer RT (2004) Patient age in Spitz nevus and malignant melanoma: implication of Bayes rule for differential diagnosis. Am J ClinPathol. 121: 872- 877.

3. Hill SJ, Delman KA (2012) Pediatric melanomas and the atypical spitzoid melanocytic neoplasms. Am J Surg 203: 761-767.

4. Argenziano G, Soyer HP (2001) Dermoscopy of pigmented skin lesions-a valuable tool for early diagnosis of melanoma. Lancet Oncol 2: 443-449.

5. Ferrara G, Argenziano G, Soyer HP (2005) The spectrum of Spitz nevi: a clinicopathologic study of 83 cases. Arch Dermatol 141: 1381-1387.

6. Pizzichetta MA, Argenziano G, Grandi G, de Giacomi C, Trevisan G, et al. (2002) Morphologic changes of a pigmented Spitz nevus assessed by dermoscopy. J Am Acad Dermatol. 47: 137-139.

7. Nino M, Brunetti B, Delfino S, Brunetti B, Panariello L, et al. (2009) follow-up study of 8 cases of childhood starburst type and proposal for management. Dermatology 218: 48-51. 